УДК 514.182 .7

DOI https://doi.org/10.32782/2664-0406.2020.37.5

\title{
Мостовенко А.В.
}

докторант кафедры начертательной геометрии и инженерной графики, Киевский национальный университет строительства и архитектуры, г. Киев

\section{МОДЕЛИРОВАНИЕ ФИЗИЧЕСКОГО ПОЛЯ ПРИ ИЗВЕСТНЫХ ЭКСПЕРИМЕНТАЛЬНО ПОЛУЧЕННЫХ ЗНАЧЕНИЯХ ПОТЕНЦИАЛОВ ОТДЕЛЬНЫХ ЕГО ТОЧЕК}

\begin{abstract}
Анотащія. На стадї архітектурного проектування будівель, споруд, а також просторів всередині та зовні об'єктів, що проектуються, однією з важливих задач є задача енергозбереження. 3 геометричної точки зору прикладами таких задач можуть бути моделювання фізичного поля від джерел енергї різного виду, визначення потенщіалу енергї в конкретній заданій точщі фізичнго поля при заданих джерелах енергї $i$ m. д. Особливу чікавість для практики становить низка зворотних задач, в яких, наприклад, за заданими параметрами окремих точок фізичного поля визначаються параметри джерел енергіi.

У цій статті запропоновано геометричний апарат, що дає змогу за відомими експериментально отриманими значеннями окремих точок фізичного поля, яке утворено в тривимірному просторі одним точковим джерелом енергї, визначати потенціал довільної точки цього поля, а також потужність точкового джерела енергії, параметри положення якого задані.

Ключові слова: геометричне моделювання, фізичне поле, потенціал енергії, точкове джерело енергї, відстань, функиія, точка, гіпербола, парабола.
\end{abstract}

Постановка проблемы. На стадии архитектурного проектирования зданий, сооружений, а также пространств внутри и снаружи проектируемых объектов одной из важных задач является задача энергосбережения. $\mathrm{C}$ геометрической точки зрения примерами таких задач могут быть моделирование физического поля от источников энергии разного вида; определение потенциала энергии в конкретной заданной точке физического поля при заданных источниках энергии и т. д. Особый интерес для практики представляет ряд обратных задач, в которых, например, по заданным параметрам отдельных точек физического поля определяются параметры источников энергии.

Анализ последних исследований. Авторами работ [3; 4] решались подобные задачи определения потенциалов точек методами непрерывной или дискретной интерполяции. Но авторами этих работ не учитывалось влияние расстояний от точек физического поля до источников энергии на потенциал поля. В работе [5] авторами предложен метод определения потенциала энергии в виде количества тепла от источника, напоминающего факел, который представлен в виде эллипсоида вращения.
Цель статьи - создание геометрического аппарата, позволяющего по известным экспериментально полученным значениям отдельных точек физического поля, которое образовано в трехмерном пространстве одним точечным источником энергии, определять потенциал произвольной точки этого поля, а также мощность точечного источника энергии, параметры положения которого заданы.

Результаты исследований. Пусть в трехмерном пространстве заданы экспериментально полученные значения потенциалов $U_{1}$, $U_{2}, U_{3}, \ldots, U_{n}$ точек физического поля, которые находятся на расстояниях $l_{1}, l_{2}, l_{3}, \ldots, l_{n}$ от точечного источника энергии соответственно. Необходимо определить мощность точечного источника энергии, который имеет ограниченную зону действия, и вывести зависимость, позволяющую определять потенциал произвольной точки этого поля.

На потенциал произвольной точки пространственного физического поля, образованного точечным источником энергии, влияет расстояние от этой точки до заданного источника энергии. С увеличением указанного расстояния потенциал в точке поля уменьшается, а с уменьшением его - увеличивается. 
Такое влияние расстояния между точкой поля и точечным источником энергии на потенциал этого поля может быть описано некоторым параметром $t$ [1].

Для решения поставленной задачи можно использовать принцип определения параметра $t$ по обобщенной схеме (рис. 1), заменив линии $m$ или $q$ некоторой кривой, которая проходит через точки, соответствующие экспериментальным данным [6]. Абсциссы заданных точек этой кривой соответственно равны $x_{i}=l_{i}$, а ординаты $y_{i}-$ неизвестны. Ординаты этих точек необходимо выразить через экспериментально полученные параметры $U_{1}, U_{2}, U_{3}, \ldots, U_{n}$.

Потенциал произвольной точки поля определяется по формуле [1]:

Потенциал произвольной точки поля определяется по формуле [1]:

$$
U_{i}=U^{*} \cdot t_{i}
$$

где $U^{*}-$ потенциал точечного источника энергии.

В соответствии со схемой (рис. 1):

$$
t_{i}=1-y_{i} .
$$

Тогда можно записать систему уравнений:

$$
\begin{aligned}
& U_{1}=U^{*}\left(1-y_{1}\right) ; \\
& U_{2}=U^{*}\left(1-y_{2}\right) ; \\
& \cdots \cdots \cdots \cdots \cdots \cdots \cdots \cdots \cdots \cdots \cdots \cdots \\
& U_{n}=U^{*}\left(1-y_{n}\right) .
\end{aligned}
$$

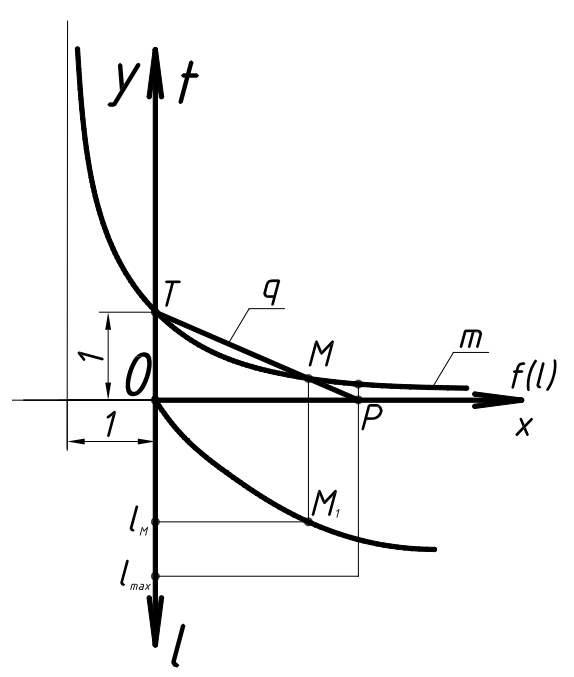

Рис. 1

Неизвестными в этой системе уравнений являются $U^{*}$ и $y_{1}, y_{2}, \ldots, y_{n}$. Число неизвестных на единицу превышает число уравнений.
Свяжем координаты заданных точек параболической зависимостью:

$$
y=a_{1} l+a_{2} l^{2}+a_{3} l^{3}+\ldots+a_{n} l^{n},
$$

где число параметров аi на единицу меньше числа экспериментально полученных точек.

Для определения параметров $a_{\mathrm{i}}$ необходимо в уравнение (3) подставить поочередно координаты известных точек. Тогда к системе уравнений (2) добавится другая система уравнений:

$$
\begin{aligned}
& y_{1}=a_{1} l_{1}+a_{2} l_{1}^{2}+a_{3} l_{1}^{3}+\ldots+a_{n} l_{1}^{n} ; \\
& y_{2}=a_{1} l_{2}+a_{2} l_{2}^{2}+a_{3} l_{2}^{3}+\ldots+a_{n} l_{2}^{n} \text {; } \\
& y_{m}=a_{1} l_{m}+a_{2} l_{m}^{2}+a_{3} l_{m}^{3}+\ldots+a_{n} l_{m}^{n},
\end{aligned}
$$

где число неизвестных параметров $a_{i}$ на единицу меньше числа уравнений.

При подстановке уі из системы (4) в систему (2) получаем новую систему уравнений, где число неизвестных соответствует числу уравнений:

$$
\begin{aligned}
& U_{1}=U^{*}\left(1-a_{1} l_{1}-a_{2} l_{1}^{2}-\ldots-a_{n} l_{1}^{n}\right) ; \\
& U_{2}=U^{*}\left(1-a_{1} l_{2}-a_{2} l_{2}^{2}-\ldots-a_{n} l_{2}^{n}\right) \text {; } \\
& U_{m}=U^{*}\left(1-a_{1} l_{m}-a_{2} l_{m}^{2}-\ldots-a_{n} l_{m}^{n}\right) .
\end{aligned}
$$

В результате решения системы (5) определяются параметры $U^{*}$ и $a_{i}$.

При подстановке уже известных параметров аі получаем уравнение параболы пго порядка, которая позволяет определить ординату $y$ при произвольно заданной величине $l_{\max } \geq l \geq 0$.

Величина $l_{\max }$ получается при пересечении параболы (3) с прямой $y=1$.

Пример.

Пусть экспериментально полученными данными являются параметры трех точек физического поля: $l_{1}=2$ лин. ед.; $l_{2}=5$ лин. ед.; $l_{3}=7$ лин. ед.; $U_{1}=7,5$ усл. ед.; $U_{2}^{2}=5$ усл. ед.; $U_{3}=2,5$ усл. ед.

Системы уравнений (4) и (5) при заданных исходных данных принимают вид: 


$$
\begin{aligned}
& y_{1}=2 a_{1}+4 a_{2} ; \\
& y_{2}=5 a_{1}+25 a_{2} ; \\
& y_{3}=7 a_{1}+49 a_{2} ; \\
& 7.5=U^{*}\left(1-2 a_{1}-4 a_{2}\right) ; \\
& 5=U^{*}\left(1-5 a_{1}-25 a_{2}\right) ; \\
& 2.5=U^{*}\left(1-7 a_{1}-49 a_{2}\right) .
\end{aligned}
$$

Результатом решения системы (6) являются ординаты точек, через которые проходит парабола (3): $y_{1}=0.1 ; y_{2}=0.4 ; y_{3}=0.7$; параметры $a_{1}=0.03 ; a_{2}=0.01$ и мощность точечного источника энергии $U^{*}=8.3333$.

Парабола (3) имеет вид:

$$
y=0.03 l+0.01 l^{2} .
$$

Откуда можно определить величину $l_{\max }$ при $y=1$ :

$$
l_{\max }=8.612
$$

и величину параметра $1 \geq t \geq 0$ в пределах $8.612 \geq l \geq 0$ для любой точки физического поля.

Изоповерхностями этого поля являются концентрические сферы с центром, совпадающим с точечным источником энергии. В сферической системе координат с центром, совпадающим с точечным источником энер-

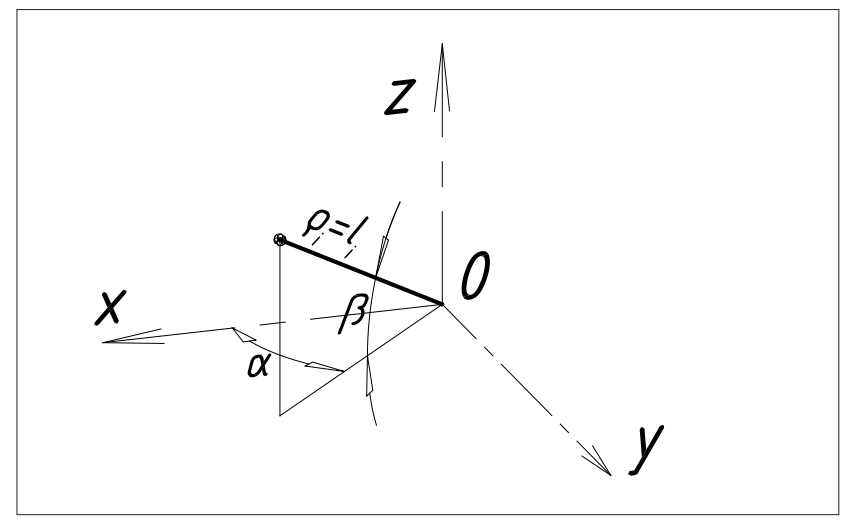

Рис. 2

гии (рис. 2), для каждого из трех заданных расстояний $l_{\mathrm{i}}$, заданным является только радиус-вектор $\rho=l_{i}$. Остальные параметры $\alpha$ и $\beta$ остаются свободными и могут принимать различные значения.

Выводы. В данной статье создан геометрический аппарат, позволяющий по известным экспериментально полученным значениям отдельных точек физического поля, которое образовано в трехмерном пространстве одним точечным источником энергии, определять потенциал произвольной точки этого поля, а также мощность точечного источника энергии, параметры положения которого заданы.

\section{Jumepamypa}

1. Мостовенко О.В. Порівняльний аналіз графіків потенціалів енергії при різних функціях від відстані. Сучасні проблеми архітектури та містобудування : Наук.-техн. Збірник. Київ, КНУБА, 2019. Вип. 53. C. 297-304.

2. Элементарный учебник физики. Учебное пособие. В 3-х т. / Под ред. Г.С. Лансберга. Т. ІІІ. Колебания и волны. Оптика. Атомная и ядерная физика. 10-е изд., перераб. Москва : Наука. Главная редакция физико-математической литературы, 1986. 656 с.

3. Сергейчук О.В. Геометричне моделювання фізичних процесів при оптимізації форми енергоефективних будинків : дис. ...д. техн. Наук : 05.01.01. Київ : КНУБА, 2008. 425 с.

4. Скочко В.І. Спеціальні геометричні моделі процесів, що розвиваються в суцільному середовищі : дис. ... к. техн. Наук : 05.01.01. Київ : КНУБА, 2012. 269 с.

5. Попов В.М., Куценко Л.М., Семенова-Куліш В.В. Метод оцінки теплового потоку, що випромінюється еліпсоїдом як факелом полум'я. Харків : ХІПБ МВС України, 2000. 144 с.

6. Мостовенко А.В. Обобщение схем для определения параметра учета влияния расстояния от точки физического поля до точечного источника энергии. Прикладна геометрія та інженерна графіка : Міжвідомчий наук.-техн. збірник. Київ : КНУБА, 2020. Вип. 98.

\section{References}

1. Elementarnyi uchebnyk fyzyky. Uchebnoe posobye. V 3-kh t. / Pod red. H.S. Lansberha. T. III. Kolebanyia y volnы. Optyka. Atomnaia y yadernaia fyzyka. 10-e yzd., pererab. M.: Nauka. Hlavnaia redaktsyia fyzykomatematycheskoi lyteraturы, 1986. 656 s. (in Russian)

2. Serheichuk O.V. Heometrychne modeliuvannia fizychnykh protsesiv pry optymizatsii formy enerhoefektyvnykh budynkiv : Dys. ...d. tekhn. Nauk : 05.01.01. K.: KNUBA, 2008. 425 s. (in Ukrainian)

3. Skochko V.I. Spetsialni heometrychni modeli protsesiv, shcho rozvyvaiutsia v sutsilnomu seredovyshchi : dys. ... k. tekhn. Nauk : 05.01.01. K.: KNUBA, 2012. 269 s. (in Ukrainian) 
4. Popov V.M., Kutsenko L.M., Semenova-Kulish V.V. Metod otsinky teplovoho potoku, shcho vyprominiuietsia elipsoidom yak fakelom polumia. Kharkiv : KhIPB MVS Ukrainy, 2000. 144 s. (in Ukrainian)

5. Mostovenko A.V. Obobshchenye skhem dlia opredelenyia parametra ucheta vlyianyia rasstoianyia ot tochky fyzycheskoho polia do tochechnoho ystochnyka ynerhyy. Prykladna heometriia ta inzhenerna hrafika: Mizhvidomchyi nauk.-tekhn. zbirnyk. K., KNUBA, 2020. Vyp. 98. (in Russian)

6. Mostovenko O.V. Porivnialnyi analiz hrafikiv potentsialiv enerhii pry riznykh funktsiiakh vid vidstani. Suchasni problemy arkhitektury ta mistobuduvannia : Nauk.-tekhn. zbirnyk. K., KNUBA, 2019. Vyp. 53. S. 297-304. (in Ukrainian).

\title{
DETERMINATION OF THE POTENTIAL OF AN ARBITRARY POINT OF PHYSICAL FIELD AT KNOWN VALUES OF POTENTIALS OF INDIVIDUAL POINTS OF PHYSICAL FIELD
}

\begin{abstract}
At the stage of architectural design of buildings, structures, as well as spaces inside and outside the designed objects, one of the important tasks is the task of energy conservation. From a geometric point of view, examples of such tasks can be: modeling a physical field from various types of energy sources; determination of the energy potential at a specific given point of a physical field for given energy sources, etc. Of particular interest for practice is a number of inverse problems in which, for example, the parameters of energy sources are determined from the given parameters of individual points of the physical field.

The potential of an arbitrary point of a physical field formed in three-dimensional space is affected by the distance of this point from the energy source. This effect decreases with increasing distance from the field point to the energy source and increases with decreasing the specified distance. Formally, this influence is described by some parameter $t$ [1].

Also, a special influence on the propagation of a physical field in three-dimensional space, and therefore on the potentials of its points, has the form of radiated energy and the medium in which this field arises. It should be noted that the energy sources that create the physical field can be point, extended (linear) [2], as well as in the form of surfaces (planes).

This article proposes a geometric apparatus that allows one to determine the potential of an arbitrary point of this field, as well as the power of a point source of energy, the position parameters of which are set from known experimentally obtained values of individual points of a physical field that is formed in three-dimensional space by a single point source of energy.

Key words: geometric modeling, physical field, energy potential, point source of energy, distance, function, point, hyperbola, parabola.
\end{abstract}

\section{Mostovenko O.V.}

Doctoral Candidate at the Department of Descriptive Geometry and Engineering Drawing,

Kyiv National University of Construction and Architecture, Kyiv 